\title{
First identification and genotyping of Enterocytozoon bieneusi in humans in Myanmar
}

\author{
Yujuan Shen ${ }^{1 \dagger}$, Baiyan Gong ${ }^{2 \dagger}$, Xiaohua Liu ${ }^{2}$, Yanchen $W^{2}{ }^{2}$, Fengkun Yang ${ }^{2}$, Jie Xu' ${ }^{1}$ Xiaofan Zhang ${ }^{1}$, \\ Jianping $\mathrm{CaO}^{1 *}$ (D) and Aiqin Liu ${ }^{2^{*}}$
}

\begin{abstract}
Background: Intestinal pathogen infections are widespread among impoverished populations. Enterocytozoon bieneusi is the most common pathogen of intestinal microsporidian species in humans worldwide. However, no epidemiological information is available on E. bieneusi infection in humans in Myanmar. The present study comprised the first identification and genotyping of E. bieneusi in humans conducted in Myanmar.

Results: A total of 172 fecal specimens were collected from the Wa people (one each) in four villages of Pangsang Township of the Matman District of Shan State, Myanmar, and each participant completed a questionnaire. E. bieneusi was identified and genotyped using polymerase chain reaction (PCR) and sequence analysis of the internal transcribed spacer (ITS) region of the ribosomal RNA (rRNA) gene. The average prevalence of E. bieneusi was $8.72 \%$ (15/172), ranging from 3.85 to $13.89 \%$ by village. E. bieneusi infection was not related to any of the risk factors studied. Six genotypes were identified, comprising two known genotypes Peru6 $(n=10)$ and $D(n=1)$ and four novel genotypes (MMR23, MMR25, MMR86, and MMR87) (one each), and two people infected with genotype Peru6 were from the same family. A phylogenetic analysis based on a neighbor-joining tree of the ITS sequences of $E$. bieneusi indicated that all the six genotypes were clustered into group 1.

Conclusions: This is the first identification and genotyping of E. bieneusi in humans in Myanmar. The observations that the two people infected with genotype Peru6 were from the same family, and that all six genotypes obtained in the present study fell into zoonotic group 1, showed the potential for anthropogenic and zoonotic transmissions. The present data argue for the importance of epidemiological control and prevention from medical sectors.
\end{abstract}

Keywords: Enterocytozoon bieneusi, Humans, Prevalence, Genotype, Phylogeny

\section{Background}

Microsporidia comprise a diverse group of unicellular and obligate intracellular parasitic fungi and have been found in a wide range of vertebrate and invertebrate hosts [1]. To date, there are about 1500 species in over 200 genera formally described, and 17 species have been found in humans with Enterocytozoon bieneusi being the

\footnotetext{
*Correspondence: caojp@yahoo.com; liuaiqin1128@126.com

${ }^{\dagger}$ Yujuan Shen and Baiyan Gong contributed equally to this work.

${ }^{1}$ National Institute of Parasitic Diseases, Chinese Center for Disease Control and Prevention, Chinese Center for Tropical Diseases Research, WHO Collaborating Center for Tropical Diseases, Key Laboratory of Parasite and Vector Biology, MOH, Shanghai 200025, China

${ }^{2}$ Department of Parasitology, Harbin Medical University, Harbin 150081, Heilongjiang, China
}

most frequently diagnosed (over $90 \%$ ) in cases of microsporidioses [2,3]. E. bieneusi is one of the most common intestinal pathogens responsible for diarrhea in infected hosts, and more seriously, chronic or life-threatening diarrhea often occur in immunodeficient or immunocompromised individuals, such as patients with acquired immune deficiency syndrome (AIDS), organ transplant recipients, patients with cancer, travelers, children, and the elderly, showing the clinical features of opportunistic infection [4]. The increasing number of cases of asymptomatic infection of E. bieneusi in immunocompetent people highlights the epidemiological importance and significance that they play in the transmission of the microorganism [5]. The identification of E. bieneusi in

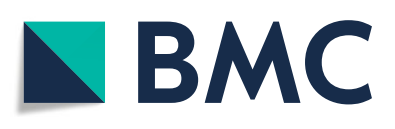

(c) The Author(s). 2020 Open Access This article is distributed under the terms of the Creative Commons Attribution 4.0 International License (http://creativecommons.org/licenses/by/4.0/), which permits unrestricted use, distribution, and reproduction in any medium, provided you give appropriate credit to the original author(s) and the source, provide a link to the Creative Commons license, and indicate if changes were made. The Creative Commons Public Domain Dedication waiver (http://creativecommons.org/publicdomain/zero/1.0/) applies to the data made available in this article, unless otherwise stated. 
numerous mammal and bird species indicates the zoonotic nature of this disease [6]. An unusual genotype, Peru16, was found in seven guinea pigs and a 2-year-old child in the same household [7]. Of course, humans could also acquire E. bieneusi infections through the anthroponotic route. Two studies conducted in a Thai orphanage provided evidence of person-to-person transmission of $E$. bieneusi, based on the findings that genotype A was detected in all E. bieneusi-positive fecal samples of the orphans $[8,9]$.

However, most human infections of E. bieneusi are considered to result from fecal-oral transmission of infective spores from infected hosts through contaminated food or water [3]. E. bieneusi has been detected in some foods (milk, raspberries, beans, and lettuce) $[10,11]$ and a foodborne outbreak caused by E. bieneusi was reported in Sweden in 2009 [11]. To date, no waterborne outbreaks have been reported. However, E. bieneusi spores have been detected in multiple water supplies, including irrigation waters used for crops, recreational waters, and effluents from wastewater treatment plants [2]. Contact with contaminated water is considered an important risk factor related to E. bieneusi infection in epidemiological studies [4].

E. bieneusi is a complex species with multiple genotypes. By sequence analysis of the internal transcribed spacer (ITS) region of the ribosomal RNA (rRNA) gene, at least 474 ITS genotypes have been identified, which can be phylogenetically divided into 11 groups (groups $1-11)$. Groups 1 and 2 are two large groups, composed of 314 and 94 genotypes, respectively [2]. To date, at least 106 genotypes have been identified in humans: 91 in group 1, six in group 2, two in group 5, five in group 6 , one in group 10, and one in the outlier, and 46 of them have been found in animals: 39 in group 1, five in group 2, one in group 6, and one in group 10 (Fig. 1). The genotypes in group 1 originate from a wide host range, including humans and numerous mammal and bird species, revealing low host specificity and the potential for zoonotic or cross-species transmission [2]. The genotypes in group 2 were once considered cattle-specific based on early studies [12]. However, with increasing epidemiological data for E. bieneusi, it has been observed that the genotypes in this group could be detected in other animal hosts; more importantly, certain genotypes (notably BEB4, BEB6, CHN3, I and J) have been found in humans, raising public health concern related to the zoonotic potential of this group [2].

Infectious diseases, including microsporidioses, usually affect people living in poverty, and these diseases may further promote poverty. Intestinal pathogen infections are widespread among Southeast Asia's most impoverished populations [13]. In Myanmar, Cryptosporidium, Giardia duodenalis, Entamoeba histolytica, Entamoeba coli, and Entamoeba nana have been detected in humans [14, 15]. However, no data are available on E. bieneusi infection in humans in this country. The Wa is one of the 135 officially recognized ethnic groups of Myanmar. Wa people live mostly in small villages in Shan State, close to the Chinese border, where the economy and culture are undeveloped, and villagers do not practice good hygiene habits (e.g., washing hands before eating meals). The objectives of the present study were to understand the prevalence and genotypes of E. bieneusi in humans in Myanmar, specifically in Wa people, using polymerase chain reaction (PCR) and

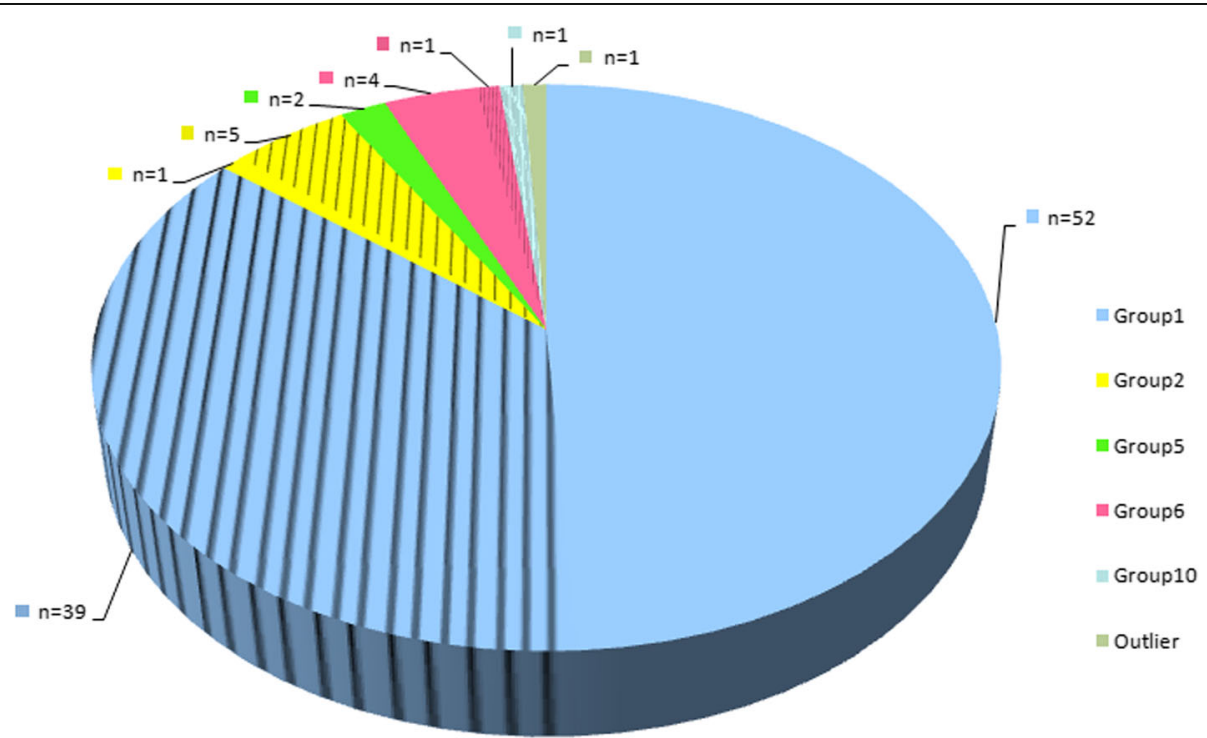

Fig. 1 Distribution of E. bieneusi genotypes in humans by group worldwide $(n=106)$. Shaded parts represent the genotypes in both humans and animals. The data presented here are based on the work of Li et al. [2] 
sequence analysis of the ITS region of the rRNA gene as well as possible risk factors related to $E$. bieneusi infection.

\section{Results}

\section{Prevalence of $E$. bieneusi}

A total of 172 fecal specimens were collected and studied for the presence of $E$. bieneusi using PCR amplification of the ITS region of the rRNA gene. A total of 15 PCR products of the expected size were confirmed positive for $E$. bieneusi by sequence analysis. The overall prevalence of $E$. bieneusi was $8.72 \%(15 / 172)$ in Wa people in the investigated areas. E. bieneusi was found in all the four investigated villages, with a prevalence ranging from 3.85 to $13.89 \%$. However, there was no statistically difference among them according to a $\chi^{2}$ test $(P>0.05)$ (Table 1$)$.

\section{Relationship of $E$. bieneusi infection and risk factors as well as gastrointestinal symptoms}

In the present study, with the help of the local Center for Disease Control and Prevention (CDC) and the people at Health Without Borders, all the participants, including 97 children (aged 7-12 years), 41 teenagers (aged 13-17 years), and 34 adults (aged > 18 years), responded to the questionnaires after they received an oral explanation of the study objectives and procedures, and provided complete information in writing. There were no significant relationships between $E$. bieneusi infection and each of the risk factors listed in Table 2 and Fig. 2. Meanwhile, the presence of E. bieneusi in fecal specimens was not related to gastrointestinal symptoms listed in Table 2. Similar results were observed about relationship of $E$. bieneusi infection and risk factors, as well as gastrointestinal symptoms, in the largest age group of children (Table 3 ).

\section{Genetic characterizations and genotype distribution of $E$. bieneusi by village}

By multiple-sequence alignment and analysis of the ITS region of the rRNA gene, 18 polymorphic sites were observed among $15 \mathrm{E}$. bieneusi isolates obtained in the present study (Table 4). Six genotypes were identified, including two known genotypes Peru6 $(n=10)$ and D $(n=1)$ and four novel genotypes (one each) named as MMR23 (GenBank: MN399816), MMR25 (GenBank:
MN399817), MMR86 (GenBank: MN399818) and MMR87 (GenBank: MN399819), which all had the largest similarity with the known genotypes Peru6 (KY950540) (99.59\%), Peru6 (KY950540) (98.77\%), GDR2 (MH714715) (99.18\%) and Henan-II (JF691565) (99.59\%), respectively. The dominant genotype, Peru6 $(66.67 \%, 10 / 15)$, could be found in all four villages. Meanwhile, two of ten people infected with genotype Peru6 were from the same family.

\section{Phylogenetic relationship of $E$. bieneusi genotypes}

In a phylogenetic analysis of the ITS sequences of $E$. bieneusi, the genetic relationship was observed among $E$. bieneusi genotypes identified in the present study. All six genotypes obtained here were clustered into zoonotic group 1: genotypes Peru6, MMR23, and MMR25 in subgroup 1b; genotypes D and MMR87 in subgroup 1a; and genotype MMR86 in subgroup 1f (Fig. 3).

\section{Discussion}

Intestinal pathogens are considered to be prevalent in areas with poor sanitation and low socioeconomic status [15]. Meanwhile, higher temperatures often increase parasite growth, reproduction and infectivity $[16,17]$. In Myanmar, there is a high annual average temperature $\left(21-32^{\circ} \mathrm{C}\right)$, resulting in the widespread presence of intestinal pathogens [15]. In the present molecular epidemiological investigation of $E$. bieneusi conducted in 172 Wa people from four villages of Pangsang Township of Matman District of Shan State, $8.72 \%$ of villagers were confirmed to be infected with E. bieneusi, with a prevalence ranging from 3.85 to $13.89 \%$ by village. The result was similar to that of a recent epidemiological study of E. bieneusi conducted in villagers in Yunnan, China, bordering with Myanmar, in which the average prevalence of $E$. bieneusi was $8.30 \%(24 / 289)$ in Yao people [5]. This similar prevalence might be related to similar climatic and socio-economic conditions on both sides of the China-Myanmar border. In fact, many factors might have influence on $E$. bieneusi infection in humans.

Immunodeficient or immunocompromised individuals, especially those associated with human immunodeficiency virus (HIV) infection and AIDS, are susceptible to infection with $E$. bieneusi. E. bieneusi has been reported to be more common in HIV-positive patients than in

Table 1 Prevalence and distribution of E. bieneusi genotypes in humans by village

\begin{tabular}{lll}
\hline Collection site & Positive no./Examined no. (\%) & Genotype/s (n) \\
\hline Village I & $4 / 32(12.50)$ & Peru6 (1), D (1), MMR23 (1), MMR25 (1) \\
Village II & $2 / 52(3.85)$ & Peru6 (2) \\
Village III & $4 / 52(7.69)$ & Peru6 (2), MMR86 (1), MMR87 (1) \\
Village IV & $5 / 36(13.89)$ & Peru6 (5) \\
Total & 15/172 (8.72) & Peru6 (10), D (1), MMR23 (1), MMR25 (1), MMR86 (1), MMR87 (1)
\end{tabular}

\footnotetext{
${ }^{a}$ The genotypes in bold are novel genotypes obtained in the present study
} 
Table 2 Analysis of risk factors for E. bieneusi infection

\begin{tabular}{|c|c|c|c|c|c|c|}
\hline \multicolumn{3}{|l|}{ Variable } & \multirow{2}{*}{$\begin{array}{l}\text { Examined no. (\%) } \\
94 / 54.65\end{array}$} & \multirow{2}{*}{$\begin{array}{l}\text { Positive no (\%) } \\
11 / 11.70\end{array}$} & \multirow{2}{*}{$\frac{\mathrm{OR}^{\mathrm{a}}\left(95 \% \mathrm{Cl}^{\mathrm{b}}\right)}{2.45(0.75,8.03)}$} & \multirow{2}{*}{$\frac{X 2 / P \text {-value }}{1.56 / 0.21}$} \\
\hline Demographic & Sex & Male & & & & \\
\hline & & Female & $78 / 45.35$ & $4 / 5.13$ & & \\
\hline & Age (years) & Children $(<13)$ & $97 / 56.39$ & $5 / 5.15$ & Ref & \\
\hline & & Teenagers (13-17) & $41 / 23.84$ & $5 / 12.20$ & $0.39(0.11,1.43)$ & $2.13 / 0.15$ \\
\hline & & Adults ( $\geq 18$ years) & $34 / 19.77$ & $5 / 14.71$ & $0.32(0.09,1.17)$ & $3.26 / 0.07$ \\
\hline \multirow{10}{*}{$\begin{array}{l}\text { Clinical } \\
\text { symptoms }\end{array}$} & Diarrhea & Yes & $42 / 24.42$ & $3 / 7.14$ & $0.76(0.20,2.82)$ & $0.01 / 0.92$ \\
\hline & & No & $130 / 75.58$ & $12 / 9.23$ & & \\
\hline & Abdominal pain & Yes & 24/13.95 & $2 / 8.33$ & $0.94(0.20,4.47)$ & $0 / 1.00$ \\
\hline & & No & $148 / 86.05$ & 13/8.78 & & \\
\hline & Nausea & Yes & $5 / 2.91$ & 0 & $1.10(1.05,1.15)$ & $-/ 1.00^{c}$ \\
\hline & & No & 167/97.09 & $15 / 8.98$ & & \\
\hline & Emesis & Yes & $8 / 4.65$ & $1 / 12.5$ & $1.53(0.18,13.35)$ & $0 / 1.00$ \\
\hline & & No & $164 / 95.35$ & $14 / 8.54$ & & \\
\hline & Anorexia & Yes & $6 / 3.49$ & $1 / 16.67$ & $2.17(0.24,19.91)$ & $0 / 1.00$ \\
\hline & & No & $166 / 96.51$ & $14 / 8.43$ & & \\
\hline \multirow{8}{*}{$\begin{array}{l}\text { Personal } \\
\text { hygiene } \\
\text { habits }\end{array}$} & Drinking boiled water & Yes & 135/78.49 & $12 / 8.89$ & $1.11(0.30,4.14)$ & $0 / 1.00$ \\
\hline & & No & $37 / 21.51$ & $3 / 8.11$ & & \\
\hline & Washing hands before meals & Yes & $98 / 56.98$ & $9 / 9.18$ & $1.15(0.39,3.38)$ & $0.06 / 0.80$ \\
\hline & & No & $74 / 43.02$ & $6 / 8.11$ & & \\
\hline & Washing hands after using toilets & Yes & $73 / 42.44$ & $9 / 12.33$ & $2.18(0.74,6.42)$ & $2.07 / 0.15$ \\
\hline & & No & $99 / 57.56$ & $6 / 6.06$ & & \\
\hline & Eating unwashed vegetables and fruits & Yes & $145 / 84.30$ & $15 / 10.34$ & $0.90(0.85,0.95)$ & $-/ 0.13^{c}$ \\
\hline & & No & $27 / 15.70$ & 0 & Ref & \\
\hline \multirow[t]{5}{*}{ Others } & Pit toilets & Public & $166 / 96.51$ & $15 / 9.04$ & $0.91(0.87,0.95)$ & $-/ 1.00^{c}$ \\
\hline & & Individual & $6 / 3.49$ & 0 & & \\
\hline & Animal feeding patterns & Free-ranging & $36 / 20.93$ & $2 / 5.56$ & $0.56(0.07,4.29)$ & 0/0.98 \\
\hline & & $\begin{array}{l}\text { Both free-ranging } \\
\text { and captive }\end{array}$ & $115 / 66.86$ & $11 / 9.57$ & $1.01(0.21,4.90)$ & $0 / 1.00$ \\
\hline & & Captive & $21 / 12.21$ & $2 / 9.52$ & Ref & \\
\hline
\end{tabular}

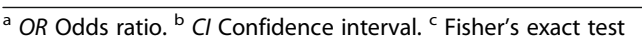

HIV-negative patients, such as $5.0 \%$ versus $1.7 \%$ in India [18] and $7.0 \%$ versus $5.1 \%$ in Portugal [19]. A high prevalence of $E$. bieneusi has also been found in HIVpositive patients, such as $27.3 \%$ in Thailand, $11.58 \%$ in China and $9.5 \%$ in India [20-22]. Children have been identified as a population group at risk for E. bieneusi infection because of immature immune system and lack of good hygienic habits [19]. A high prevalence of E. bieneusi has been reported in non-diarrheal children (9.3\%) in Nigeria [23], and in diarrheal children (22.5\%) in China [24]. In Uganda, HIV-positive children were observed to be more likely to have E. bieneusi in their stool than HIV-negative children (76.9\% versus 6.6\%) [25]. Some studies reported a high prevalence of $E$. bieneusi in elderly persons (17.0\%) and in cancer patients (40\%)
[26, 27]. In addition, poor personal hygiene habits possibly increase the risk of $E$. bieneusi infection. In China, the HIV/AIDS patients who drink unboiled water and/or do not wash hands before meals showed a statistically higher prevalence of $E$. bieneusi than the other patients $(P<0.05)[22,28]$. However, in the present study, no correlation was found between $E$. bieneusi infection and any of risk factors analyzed. Similarly, statistical calculations showed that none of the gastrointestinal symptoms recorded was related to E. bieneusi infection. Reported symptoms might be caused by other intestinal pathogens. Indeed, the appearance and severity of clinical symptoms are affected by multiple factors. In addition to the immune status of the infected hosts, intestinal pathogen species and their genetic background as well 


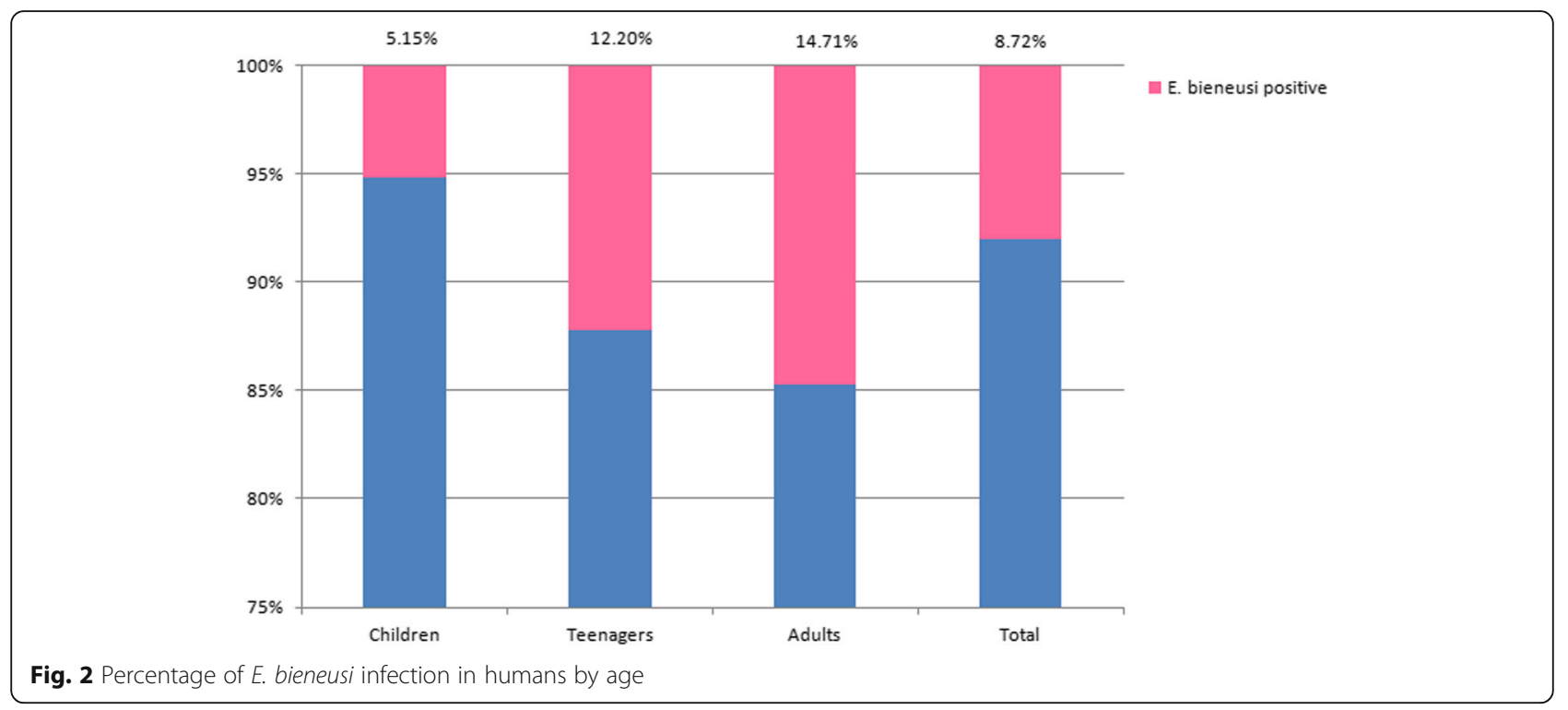

as the infection intensity in humans, could make differences in clinical symptoms. Even changes in diet can affect fecal consistency (e.g., the presence or absence of diarrhea). Therefore, future work will focus on the relationship between $E$. bieneusi infection and clinical symptoms, and the interaction of intestinal pathogens, including $E$. bieneusi in clinical gastrointestinal symptoms.

In the present study, based on sequence analysis of the ITS region, 18 polymorphic sites were observed among 15 E. bieneusi isolates and six genotypes were identified: two known genotypes (Peru6 and D) and four novel genotypes (named as MMR23, MMR25, MMR86 and MMR87). Genotype Peru6 showed an absolute predominance $(66.67 \%, 10 / 15)$ in Wa people in the investigated areas, similar to a recent study conducted in neighboring China $(87.50 \%, 21 / 24)$ [5]. Genotype Peru6 is actually a rare genotype in humans. Since the first identification in humans in Peru in 2003 [29], this genotype has only been found in humans in Peru [7, 30, 31], Portugal [19] and China [5]. Genotype D is the most prevalent genotype detected in humans. To date, this genotype has been found in humans in four continents, including Asia (China, India, Iran, Thailand and Vietnam), South America (Brazil and Peru), Europe (Netherlands, Poland, Portugal, Russia, Spain and UK) and Africa (Cameroon, Congo, Gabon, Malawi, Niger, Nigeria, Sao Tome and Tunis) [2]. Both genotypes Peru6 and D have a wide host range of animals: Peru6 in eight and nine species of mammals and birds [5], respectively, and D in 24 and seven species of mammals and birds [2], respectively, indicating the possibility of zoonotic transmissions. In a phylogenetic analysis of the ITS sequences of E. bieneusi, the observation that all four novel genotypes (MMR23, MMR25, MMR86 and MMR87) fell into group 1 suggests the possibility of zoonotic transmission. In addition, among 10 people infected with genotype Peru6, two of them were confined to the same family, indicating the possibility of anthropogenic transmission.

The present study also has some limitations. The sample size of this ethnic group in Myanmar was very small; therefore, these results might not reflect the prevalence of $E$. bieneusi in humans in this country. The true burden of human microsporidioses caused by $E$. bieneusi needs to be assessed by systematic molecular epidemiological investigations in the future.

\section{Conclusions}

The present study is the first identification and genotyping of $E$. bieneusi in humans in Myanmar. An average prevalence of $E$. bieneusi was $8.72 \%(15 / 172)$ in Wa people in the investigated areas, and six genotypes were identified, with genotype Peru6 being dominant. The previous observation of genotypes Peru6 and D in numerous animals, and the fact of four novel genotypes (MMR23, MMR25, MMR86 and MMR87) falling into group 1 suggest the possibility of zoonotic transmission. Thus, it is necessary to carry out epidemiological investigations of $E$. bieneusi in local animals. This is an important step in adequately controlling E. bieneusi infection in humans, particularly in the absence of an effective vaccine and available drugs. Although fumagillin has been used successfully in humans to treat intestinal microsporidioses caused by E. bieneusi, its efficacy is counterbalanced by its adverse effects [32]. The possible existence of anthropogenic transmission highlights the importance of health education in people living in the investigated areas. The present data argue for the importance of epidemiological control and prevention from medical sectors. 
Table 3 Analysis of risk factors for E. bieneusi infection in children

\begin{tabular}{|c|c|c|c|c|}
\hline Variable & Examined no. (\%) & Positive no (\%) & $\mathrm{OR}^{\mathrm{a}}\left(95 \% \mathrm{Cl}^{\mathrm{b}}\right)$ & X2/P-value \\
\hline \multicolumn{5}{|l|}{ Demographic factor } \\
\hline \multicolumn{5}{|l|}{ Sex } \\
\hline Male & $44 / 45.36$ & $4 / 9.09$ & $5.20(0.56,48.35)$ & $1.29 / 0.26$ \\
\hline Female & $53 / 54.64$ & $1 / 1.89$ & & \\
\hline \multicolumn{5}{|l|}{ Clinical symptoms } \\
\hline \multicolumn{5}{|l|}{ Diarrhea } \\
\hline Yes & $26 / 26.80$ & $2 / 7.69$ & $1.89(0.30,12.00)$ & $0.03 / 0.87$ \\
\hline No & $71 / 73.20$ & $3 / 4.23$ & & \\
\hline \multicolumn{5}{|l|}{ Abdominal pain } \\
\hline Yes & $14 / 14.43$ & $1 / 7.14$ & $0.66(0.07,6.36)$ & $0 / 1.00$ \\
\hline No & $83 / 85.57$ & $4 / 4.82$ & & \\
\hline \multicolumn{5}{|l|}{ Nausea } \\
\hline Yes & $3 / 3.09$ & 0 & $1.03(1.00,1.07)$ & $-/ 1.00^{c}$ \\
\hline No & $94 / 96.91$ & $5 / 5.32$ & & \\
\hline \multicolumn{5}{|l|}{ Emesis } \\
\hline Yes & $2 / 2.06$ & 0 & $1.02(1.00,1.05)$ & $-/ 1.00^{c}$ \\
\hline No & $95 / 97.94$ & $5 / 5.26$ & & \\
\hline \multicolumn{5}{|l|}{ Anorexia } \\
\hline Yes & $2 / 2.06$ & 0 & $1.02(1.00,1.05)$ & $-/ 1.00^{c}$ \\
\hline No & $95 / 97.94$ & $5 / 5.26$ & & \\
\hline \multicolumn{5}{|l|}{ Personal hygiene habits } \\
\hline \multicolumn{5}{|l|}{ Drinking boiled water } \\
\hline Yes & $71 / 73.20$ & $2 / 2.82$ & $4.50(0.71,28.63)$ & $1.45 / 0.23$ \\
\hline No & $26 / 26.80$ & $3 / 11.54$ & & \\
\hline \multicolumn{5}{|l|}{ Washing hands before meals } \\
\hline Yes & $42 / 43.30$ & $4 / 9.52$ & $0.18(0.02,1.64)$ & $1.53 / 0.22$ \\
\hline No & $55 / 56.70$ & $1 / 1.82$ & & \\
\hline \multicolumn{5}{|l|}{ Washing hands after using toilets } \\
\hline Yes & $31 / 31.96$ & 0 & $1.08(1.01,1.16)$ & $-/ 0.17^{c}$ \\
\hline No & $66 / 68.04$ & $5 / 7.58$ & & \\
\hline \multicolumn{5}{|c|}{ Eating unwashed vegetables and fruits } \\
\hline Yes & $79 / 81.44$ & $5 / 6.33$ & $1.24(1.12,1.38)$ & $-10.58^{c}$ \\
\hline No & 18/18.56 & 0 & & \\
\hline \multicolumn{5}{|l|}{ Others } \\
\hline \multicolumn{5}{|l|}{ Pit toilets } \\
\hline Public & $95 / 97.94$ & $5 / 5.26$ & $1.02(1.00,1.05)$ & $-/ 1.00^{c}$ \\
\hline Individual & $2 / 2.06$ & 0 & & \\
\hline \multicolumn{5}{|l|}{ Animal feeding patterns } \\
\hline Free-ranging & 29/29.90 & $1 / 3.45$ & $0.39(0.02,6.85)$ & $0 / 1.00$ \\
\hline Both free-ranging and captive & $56 / 57.73$ & $3 / 5.36$ & $0.62(0.06,6.56)$ & $0 / 1.00$ \\
\hline Captive & $12 / 12.37$ & $1 / 8.33$ & Ref & \\
\hline
\end{tabular}

a OR Odds ratio. ${ }^{\mathrm{b}} \mathrm{Cl}$ Confidence interval. ${ }^{\mathrm{c}}$ Fisher's exact test 
Table 4 Variation at 18 polymorphic sites within the ITS sequences of E. bieneusi isolates in Myanmar

\begin{tabular}{|c|c|c|c|c|c|c|c|c|c|c|c|c|c|c|c|c|c|c|c|}
\hline \multirow{2}{*}{$\begin{array}{l}\text { Genotype } \\
\text { (no.) }\end{array}$} & \multirow{2}{*}{$\begin{array}{l}\text { GenBank } \\
\text { accession } \\
\text { no. }\end{array}$} & \multicolumn{18}{|c|}{ Nucleotide at position: } \\
\hline & & $\overline{12}$ & 30 & 31 & 35 & 38 & 58 & 81 & 93 & 100 & 113 & 117 & 131 & 138 & 169 & 176 & 195 & 200 & 221 \\
\hline \multicolumn{20}{|l|}{ Known $^{a}$} \\
\hline Peru6 (10) & KY950540 & G & $\mathrm{T}$ & $A$ & $\mathrm{~T}$ & A & A & C & $\mathrm{T}$ & A & C & G & G & G & G & A & G & A & A \\
\hline $\mathrm{D}(1)$ & MG491314 & G & $\mathrm{T}$ & G & $\mathrm{T}$ & G & G & C & C & G & C & $\mathrm{T}$ & G & G & G & A & G & A & A \\
\hline \multicolumn{20}{|l|}{ Novel } \\
\hline MMR23 (1) & MN399816 & G & $\mathrm{T}$ & $A$ & $\mathrm{~T}$ & A & A & $C$ & $\mathrm{~T}$ & A & C & G & G & G & A & A & G & A & A \\
\hline MMR25 (1) & MN399817 & G & $\mathrm{T}$ & $A$ & $\mathrm{~T}$ & G & A & C & $\mathrm{T}$ & A & C & G & G & G & A & A & A & A & A \\
\hline MMR86 (1) & MN399818 & $\mathrm{T}$ & C & A & $C$ & G & G & $\mathrm{T}$ & $\mathrm{T}$ & G & $\mathrm{T}$ & $\mathrm{T}$ & A & A & G & G & G & A & A \\
\hline MMR87 (1) & MN399819 & G & $\mathrm{T}$ & G & $\mathrm{T}$ & G & G & C & C & G & C & $\mathrm{T}$ & G & G & G & A & G & G & G \\
\hline
\end{tabular}

${ }^{a}$ Accession numbers of known genotypes, indicating that E. bieneusi isolates have 100\% homology with the sequences from GenBank

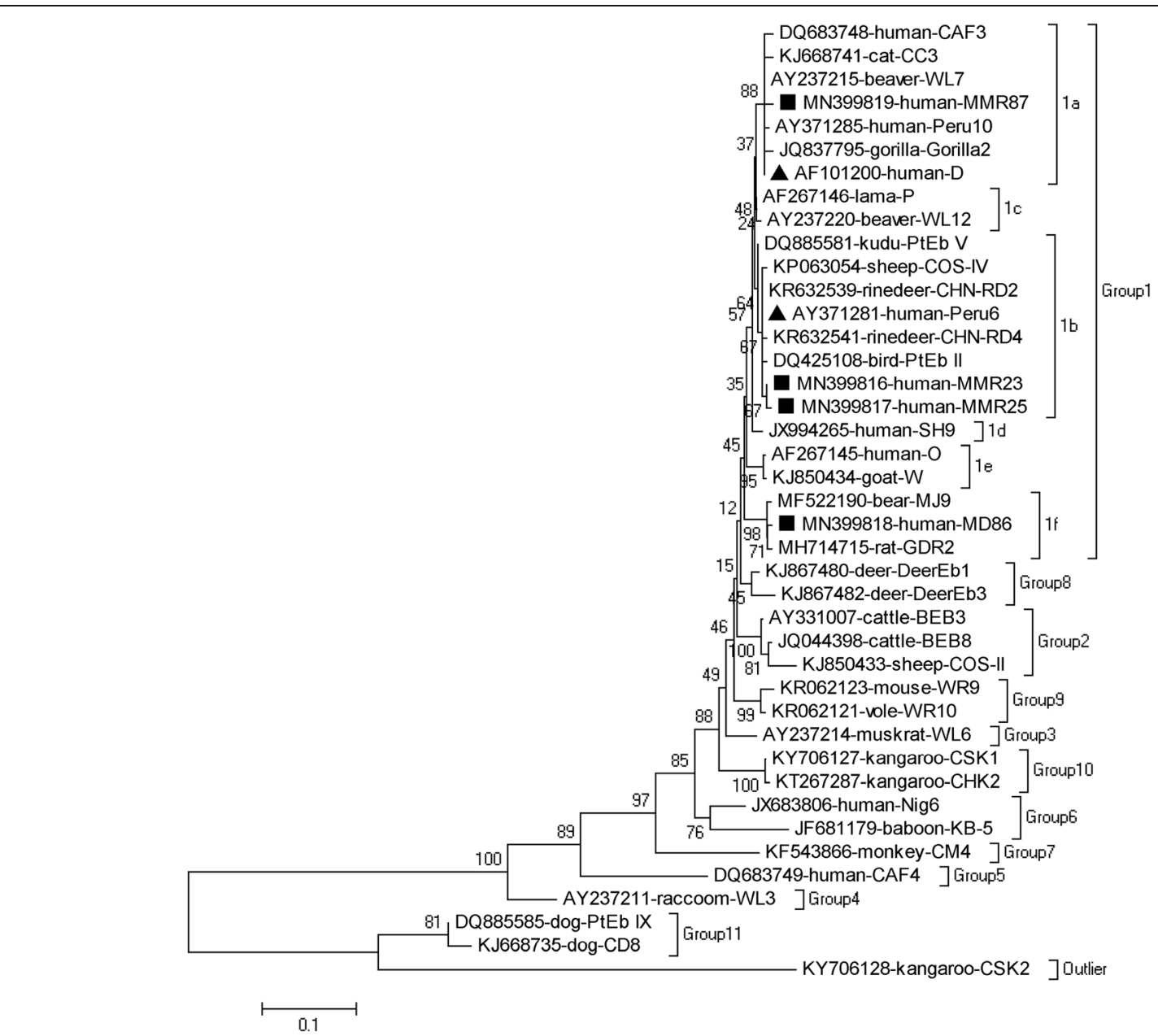

Fig. 3 Phylogenetic relationships of the genotypes of E. bieneusi. The relationships of the genotypes of E. bieneusi identified in this study and known genotypes published in GenBank were inferred using a neighbor-joining analysis of ITS sequences based on genetic distances calculated by the Kimura 2-parameter model. The numbers on the branches are percent bootstrapping values from 1000 replicates. Each sequence is identified by its accession number, host origin, and genotype designation. The group terminology for the clusters is based on the work of Li et al. [2]. The triangles and squares filled in black indicate known genotypes and novel genotypes identified in this study, respectively 


\section{Methods}

\section{Study population}

In October, 2018, fresh fecal specimens (approximately 5-10 g) were collected from 172 Wa people aged from 7 to 53 years distributing in four villages of Pangsang Township of Matman District of Shan State, Myanmar (geographical coordinates: $99.11{ }^{\circ} \mathrm{E}$ longitude, $22.10{ }^{\circ} \mathrm{N}$ latitude). All the fecal specimens were from immunocompetent individuals. Only one specimen per participant was included in the present study. Information about participants was collected and recorded by a structured questionnaire including socio-demographic characteristics, possible risk factors related to E. bieneusi infection as well as common gastrointestinal symptoms. The specimens were transported to the laboratory in a cooler with ice packs within $24 \mathrm{~h}$ after collection and stored in refrigerators at $-20{ }^{\circ} \mathrm{C}$ before DNA extraction.

\section{DNA extraction}

Genomic DNA of E. bieneusi was directly extracted from 180 to $200 \mathrm{mg}$ of each of 172 fecal specimens using a QIAamp DNA stool mini kit (QIAgen 51,504, Hilden, Germany) according to the manufacturer's instructions. All the reagents were provided by the manufacturer. To obtain a high yield of DNA, the lysis temperature was increased to $95^{\circ} \mathrm{C}$. DNA was finally eluted in $200 \mu \mathrm{l}$ of $\mathrm{AE}$ and stored at $-20^{\circ} \mathrm{C}$ in a freezer prior to PCR analysis.

\section{PCR amplification.}

E. bieneusi was identified and genotyped by PCR of an approximately 410-bp of the rRNA gene that covered the entire ITS region (243 bp) using nested primers as described [33]. TaKaRa Taq DNA Polymerase (TaKaRa Bio Inc., Tokyo, Japan) was used for all PCR amplifications. A positive control (DNA of a sheep-derived genotype COS-IV) and a negative control (no DNA water) were included with each batch of specimens analyzed. Each specimen was analyzed at least twice. All secondary PCR products were subjected to electrophoresis in a $1.5 \%$ agarose gel and visualized under UV by staining the gel with GelStrain (TransGen Biotech., Beijing, China). Details on methods are available in Protocols.io open access repository at the following link: dx.doi.org/1 $0.17504 /$ protocols.io.yvjfw $4 n$.

\section{Nucleotide sequencing and analyzing}

All secondary PCR products of the anticipated size were sent to Comate Bioscience Company Limited (Jilin, China) for DNA sequencing on an ABI Prism $3730 \mathrm{XL}$ DNA Analyzer by Sinogeno-max Biotechnology Co., Ltd. (Beijing, China), using the Big Dye Terminator v3.1 Cycle Sequencing Kit (Applied Biosystems, USA). The sequence accuracy was confirmed by two-directional sequencing and by sequencing a new PCR product if necessary. Nucleotide sequences obtained in the present study were aligned with each other and reference sequences deposited in the GenBank database using the Sequence Basic Local Alignment Search Tool (BLAST) and Clustal X 1.83 (http://www.clustal.org/). All the genotypes were identified only based on $243 \mathrm{bp}$ of the ITS region of the rRNA gene of E. bieneusi according to the established nomenclature system [34]. The first published names of the genotypes would be given if they produced sequences identical to those previously deposited in the GenBank database. In contrast, novel genotypes producing different sequences from published ones were given genotype names by adding specimen codes behind MMR (the abbreviation of Myanmar).

\section{Phylogenetic and statistical analyses}

The genetic proximity of all the genotypes obtained in our study was compared with each other and previously reported genotypes by constructing a neighbor-joining analysis of the ITS sequences based on genetic distances calculated by the Kimura two-parameter model implemented in the program Mega 5 (http://www.megasoftware. net/). A bootstrap analysis with 1000 replicates was used to assess the reliability of the tree. A nucleotide sequence of $E$. bieneusi from a kangaroo (GenBank: KY706128) was used as outgroup in phylogenetic analysis.

The statistical analysis was performed using the Statistical Package for the Social Sciences (SPSS) 19.0. Pearson chi-square $\left(\chi^{2}\right)$ and Fisher's exact tests were used to determine the relationship between $E$. bieneusi infection and possible risk factor variables and gastrointestinal symptoms (Table 2), respectively. The level of statistical significance was set as $P<0.05$.

\section{Abbreviations \\ AIDS: Acquired immune deficiency syndrome; Cl: Confidence interval; HIV: Human immunodeficiency virus; ITS: Internal transcribed spacer;} OR: Odds ratio; PCR: Polymerase chain reaction; rRNA: Ribosomal RNA

\section{Acknowledgments}

We thank the staff at the department of helminth for Yunnan Institute of Parasitic Diseases and the Centre for Disease Control and Prevention, and Zhiliang Zheng at Health Without Borders, for their assistance with sample collection and case investigation.

\section{Authors' contributions}

JC, AL and YS conceived and designed experiments. BG and FY collected samples. YS and BG performed laboratory experiments. BG, XL and YW analyzed and interpreted data. FY, JX, XZ, YS and JC contributed reagents and materials. BG and YS wrote the manuscript. AL and JC revised the manuscript. All authors have read and approved the final version of the manuscript.

\section{Funding}

This work was supported partially by the Chinese National Science and Technology Major Project (No. 2018ZX10713001-004 to YS), and Chinese Special Program for Scientific Research of Public Health (No. 201502021 to JC), The funders had no role in the study design, data collection and analysis, the decision to publish, or preparation of the manuscript. 


\section{Availability of data and materials}

ITS sequences of four novel $E$. bieneusi genotypes obtained in the present study were deposited in the GenBank database under the following accession numbers: MN399816-MN399819.

\section{Ethics approval and consent to participate}

The present study was ethically approved by the Ethics Committee of the National Institute of Parasitic Diseases, Chinese Center for Disease Control and Prevention, China (No. 2015-11), and the Myanmar Eastern Shan State Special Region 2 Ethic Health Organization. All the participants were informed about the study objectives and procedures before the start of the study. Informed written consents were obtained from adult participants or guardians of minors under the age of 18

\section{Consent for publication}

Not applicable

\section{Competing interests}

The authors declare that they have no competing interests.

Received: 17 September 2019 Accepted: 30 December 2019

Published online: 13 January 2020

\section{References}

1. Stentiford GD, Becnel J, Weiss LM, Keeling PJ, Didier ES, Williams BP, et al. Microsporidia-emergent pathogens in the global food chain. Trends Parasitol. 2016:32:336-48.

2. Li W, Feng Y, Santin M. Host specificity of Enterocytozoon bieneusi and public health implications. Trends Parasitol. 2019;35:436-51.

3. Qiu L, Xia W, Li W, Ping J, Ding S, Liu H. The prevalence of microsporidia in China: a systematic review and meta-analysis. Sci Rep. 2019;9:3174.

4. Matos O, Lobo ML, Xiao L. Epidemiology of Enterocytozoon bieneusi infection in humans. J Parasitol Res. 2012;2012:981424.

5. Gong B, Yang Y, Liu X, Cao J, Xu M, Xu N, et al. First survey of Enterocytozoon bieneusi and dominant genotype Peru6 among ethnic minority groups in southwestern China's Yunnan Province and assessment of risk factors. PLoS Negl Trop Dis. 2019;13:e0007356.

6. Santín M, Fayer R. Microsporidiosis: Enterocytozoon bieneusi in domesticated and wild animals. Res Vet Sci. 2011;90:363-71.

7. Cama VA, Pearson J, Cabrera L, Pacheco L, Gilman R, Meyer S, et al. Transmission of Enterocytozoon bieneusi between a child and Guinea pigs. J Clin Microbiol. 2007:45:2708-10.

8. Leelayoova S, Subrungruang I, Rangsin R, Chavalitshewinkoon-Petmitr P, Worapong J, Naaglor T, et al. Transmission of Enterocytozoon bieneusi genotype a in a Thai orphanage. Am J Trop Med Hyg. 2005;73:104-7.

9. Pagornrat W, Leelayoova S, Rangsin R, Tan-Ariya P, Naaglor T, Mungthin M. Carriage rate of Enterocytozoon bieneusi in an orphanage in Bangkok. Thailand J Clin Microbiol. 2009:47:3739-41.

10. Lee $\mathrm{JH}$. Molecular detection of Enterocytozoon bieneusi and identification of a potentially human-pathogenic genotype in milk. Appl Environ Microbiol. 2008:74:1664-6.

11. Decraene V, Lebbad M, Botero-Kleiven S, Gustavsson AM, Löfdahl M. First reported foodborne outbreak associated with microsporidia, Sweden, October 2009. Epidemiol Infect. 2012;140:519-27.

12. Zhao W, Zhang W, Yang F, Zhang L, Wang R, Cao J, et al. Enterocytozoon bieneusi in dairy cattle in the northeast of China: genetic diversity of ITS gene and evaluation of zoonotic transmission potential. J Eukaryot Microbiol. 2015;62:553-60.

13. Al-Harazi T, Ghani MK, Othman H. Prevalence of intestinal protozoan infections among orang Asli schoolchildren in Pos Senderut, Pahang. Malaysia J Egypt Soc Parasitol. 2013;43:561-8.

14. Aye T, Moe K, Nyein MM, Swe T. Cryptosporidiosis in Myanmar infants with acute diarrhea. Southeast Asian J Trop Med Public Health. 1994;25:654-6.

15. Kim MJ, Jung BK, Cho J, Kim DG, Song H, Lee KH, et al. Prevalence of intestinal protozoans among schoolchildren in suburban areas near Yangon. Myanmar Korean J Parasitol. 2016:54:345-8.

16. Stude A, Thieltges D, Poulin R. Parasites and global warming: net effects of temperature on an intertidal host-parasite system. Mar Ecol Prog Ser. 2010;415:11-22.
17. Macnab V, Barber I. Some (worms) like it hot: fish parasites grow faster in warmer water, and alter host thermal preferences. Glob Change Biol. 2012; 18:1540-8.

18. Kaushik S, Saha R, Das S, Ramachandran VG, Goel A. Pragmatic combination of available diagnostic tools for optimal detection of intestinal microsporidia. Adv Exp Med Biol. 2018;1057:85-94.

19. Lobo ML, Xiao L, Antunes F, Matos O. Microsporidia as emerging pathogens and the implication for public health: a 10-year study on HIV-positive and -negative patients. Int J Parasitol. 2012;42:197-205.

20. Wanachiwanawin D, Manatsathit S, Lertlaituan P, Thakerngpol K, Suwanagool P. Intestinal microsporidiosis in HIV infected patients with chronic diarrhea in Thailand. Southeast Asian J Trop Med Public Health. 1998:29:767-71.

21. Kumar SS, Ananthan S, Joyee AG. Detection of Enterocytozoon bieneusi (microsporidia) by polymerase chain reaction (PCR) using species-specific primer in stool samples of HIV patients. Indian J Med Res. 2005;121:215-9.

22. Liu H, Jiang Z, Yuan Z, Yin J, Wang Z, Yu B, et al. Infection by and genotype characteristics of Enterocytozoon bieneusi in HIV/AIDS patients from Guangxi Zhuang autonomous region. China BMC Infect Dis. 2017;17:684.

23. Ayinmode AB, Ojuromi OT, Xiao L. Molecular identification of Enterocytozoon bieneusi isolates from Nigerian children. J Parasitol Res. 2011; 2011:129542.

24. Zhang X, Wang Z, Su Y, Liang X, Sun X, Peng S, et al. Identification and genotyping of Enterocytozoon bieneusi in China. J Clin Microbiol. 2011;49: 2006-8

25. Tumwine JK, Kekitiinwa A, Bakeera-Kitaka S, Ndeezi G, Downing R, Feng X, et al. Cryptosporidiosis and microsporidiosis in Ugandan children with persistent diarrhea with and without concurrent infection with the human immunodeficiency virus. Am J Trop Med Hyg. 2005;73:921-5.

26. Lores B, López-Miragaya I, Arias C, Fenoy S, Torres J, Del-Aguila C. Intestinal microsporidiosis due to Enterocytozoon bieneusi in elderly human immunodeficiency virus--negative patients from Vigo. Spain Clin Infect Dis. 2002:34:918-21.

27. Jiménez-González GB, Martínez-Gordillo MN, Caballero-Salazar S, Peralta-Abarca GE, Cárdenas-Cardoz R, Arzate-Barbosa P, et al. Microsporidia in pediatric patients with leukemia or limphoma. Rev Investig Clin. 2012;6:25-31.

28. Wang L, Zhang H, Zhao X, Zhang L, Zhang G, Guo M, et al. Zoonotic Cryptosporidium species and Enterocytozoon bieneusi genotypes in HIVpositive patients on antiretroviral therapy. J Clin Microbiol. 2013;51:557-63.

29. Sulaiman IM, Bern C, Gilman R, Cama V, Kawai V, Vargas D, et al. A molecular biologic study of Enterocytozoon bieneusi in HIV-infected patients in Lima, Peru. J Eukaryot Microbiol. 2003;50(Suppl):591-6.

30. Bern C, Kawai V, Vargas D, Rabke-Verani J, Williamson J, Chavez-Valdez R, et al. The epidemiology of intestinal microsporidiosis in patients with HIV/ AIDS in Lima. Peru J Infect Dis. 2005;191:1658-64.

31. Feng Y, Li N, Dearen T, Lobo ML, Matos O, Cama V, et al. Development of a multilocus sequence typing tool for high-resolution genotyping of Enterocytozoon bieneusi. Appl Environ Microbiol. 2011;77:4822-8.

32. Anane S, Attouchi H. Microsporidiosis: epidemiology, clinical data and therapy. Gastroent Clin Biol. 2010;34:450-64.

33. Mirjalali H, Mirhendi H, Meamar AR, Mohebali M, Askari Z, Mirsamadi ES, et al. Genotyping and molecular analysis of Enterocytozoon bieneusi isolated from immunocompromised patients in Iran. Infect Genet Evol. 2015;36:244-9.

34. Santín M, Fayer R. Enterocytozoon bieneusi genotype nomenclature based on the internal transcribed spacer sequence: a consensus. J Eukaryot Microbiol. 2009:56:34-8.

\section{Publisher's Note}

Springer Nature remains neutral with regard to jurisdictional claims in published maps and institutional affiliations. 